\title{
Development of surgical protocol for implantation of tracheal prostheses in sheep
}

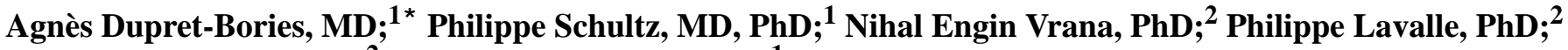 \\ Dominique Vautier, PhD; ${ }^{2}$ Christian Debry, MD, PhD $^{1}$ \\ ${ }^{1}$ Department of Otorhinolaryngology and Technical Research Team, Hôpitaux Universitaires de Strasbourg, Hôpital \\ de Hautepierre, France; ${ }^{2}$ Department of Research, Institut National de la Santé et de la Recherche Médicale \\ (INSERM), INSERM Unité 977, Strasbourg, France
}

\begin{abstract}
This article documents experiments performed in ewes to design an artificial larynx. The artificial larynx is composed of a hollow, porous tube that elongates the trachea and is capped with a valve that acts as a laryngeal sphincter. Through an industrial collaboration, our team developed a porous biomaterial that can be colonized by cervical tissues. This biomaterial has been used in animals to replace part of the trachea, but it is meant to eventually substitute for laryngeal cartilage. The tracheal prosthesis is a hollow cylindrical tube composed of titanium microbeads. We performed a study in large animals to establish an optimal surgical protocol for tracheal replacement in humans. The study included 11 sheep $(n=11)$ and compared 5 methods of implantation. We successfully established an optimal three-step surgical protocol to make the porous-titanium tracheal prosthesis functional: (1) large lumen endoprosthetics, (2) colonization by the peripheral tissues, and (3) endoprosthetic epithelialization. This study is the first step in developing an artificial larynx because it successfully identifies a biomaterial capable of extending the trachea to allow it to open at the junction of the upper aerodigestive tracts.
\end{abstract}

Key words: artificial larynx, epithelium, function, laryngeal cancer, laryngectomees, outcome, porous titanium, prosthetic surgery, rehabilitation, sheep, surgical protocol, survival rate.

\section{INTRODUCTION}

Extensive squamous cell carcinoma of the larynx and pharynx could necessitate performing a total laryngec- tomy. More than 57,000 laryngectomees live in the United States, according to the International Association of Laryngectomees (http://www.theial.com). The laryngectomy results in severe mutilation because it requires the anastomosis of the trachea to the skin and the complete separation of the respiratory and digestive tracts. The disability resulting from the loss of the larynx is a public health problem [1]. It leads to significant difficulties with social reintegration, because only palliative measures are available for vocal rehabilitation and the tracheostomy must be maintained for life.

For several years, our team has been interested in developing an artificial larynx. This would require creating a permanent, rigid, biointegrable structure [2-4] to replace the laryngotracheal tract. This structure, which is designed to replace a tracheal segment, must be sufficiently rigid to support the proximal insertion of a neosphincter that opens during respiration and closes

Abbreviations: $\mathrm{IM}=$ implantation method, $\mathrm{IMM}=$ Institut Mutualiste Monsouris, INSERM = Institut National de la Santé et de la Recherche Médicale.

* Address all correspondence to Agnès Dupret-Bories, MD; Department of Otorhinolaryngology and Technical Research Team, CHU Hautepierre, avenue Molière, 67098 Strasbourg, Cedex, France; +03-88-127654; fax: +03-88-127656.

Email: agnes.dupret@chru-strasbourg.fr

DOI:10.1682/JRRD.2010.10.0194 
during the passage of a food bolus and, at best, would include phonatory capabilities. Laryngeal tissue is comparable to tracheal tissue, except for the vocal cords; the outer layer of the vocal cords consists of perichondrium and connective tissue, and the inner layer contains cartilage covered with mucosal epithelium consisting mainly of pseudostratified ciliated cells. Tracheal reconstructions are a step toward laryngeal reconstructions.

The restoration of laryngeal function would begin with the use of an entirely integrated biomaterial to extend the proximal tracheal segment remaining after a total laryngectomy. We chose titanium because it is routinely used in orthopedic and maxillofacial surgery for its biomechanical properties and electrochemical stability [5]. Moreover, titanium is an inert and nontoxic metal that is insensitive to bacteria and mold. Titanium's rigidity provides support when it is replacing multiple tracheal rings. A few years ago, our team designed a porous biomaterial composed of titanium microbeads. The pores between the microbeads promote the material's integration by allowing cellular colonization from surrounding tissues. We have thereby succeeded in developing the hollow tube needed to extend or replace part of the trachea. After the trachea is resected and the prosthesis is placed, the tissues surrounding the prosthesis must fill the pores in the biomaterial and provide a vascular network to nourish and oxygenate the regenerating endoluminal epithelium from the tracheal extremities.

In an initial study, Schultz et al. implanted tracheal prostheses made from porous titanium in rats that were rapidly integrated by vascularized colonizing tissue and later by endoluminal epithelial tissues [2]. After healing, more than 50 percent of the animals survived until scheduled euthanasia between 3 months and 1 year later [2-3]. These data encouraged us to pursue testing in sheep, whose tracheal diameter is very close to that of humans [6]. An initial short-term (6-month) study using six animals was conducted and resulted in well-integrated material but with a low survival rate [4]. Implanting the prostheses in a single step, without an endoluminal silicone tube (the same operative protocol that was used in the rat study just mentioned), led to stenosis of the tracheo-prosthetic anastomoses with little endoprosthetic epithelialization.

These findings led us to research an optimal operative protocol to improve the impermeability and endoluminal colonization of the prostheses. To this end, we implanted the prostheses in sheep using five different methods (described in the next section) and compared their survival rates and histological results. We analyzed the colonization of porous titanium prostheses with and without conditioning (placing the prosthesis in muscle for colonization before implantation) and the use of a silicone tube for endoprosthetic calibration. We also assessed the improvement in the prostheses' endoluminal epithelialization via the application of an endoprosthetic mucous graft. This study compares and analyzes the clinical and histological results of these five surgical techniques. Our aim was to determine the ideal surgical procedure for replacing a tracheal segment with a porous titanium prosthesis in large animals before the procedure is potentially translated to humans.

\section{METHODS}

\section{Manufacturing Prostheses}

The tracheal prostheses (Figure 1) were manufactured by PROTiP (Strasbourg, France). They were 400 to $500 \mu \mathrm{m}$ in diameter and formed from a mixture of titanium beads [4]. The titanium was manufactured according to the Association française de normalisation (French Association of Normalization) standards for use of Ti40 as a surgical material. We placed the beads in a mold, then welded them together by a discharge current. The pore size between each bead was approximately $150 \mu \mathrm{m}$, i.e., a porosity of about 35 percent. The prostheses were composed of four layers of beads fused into the shape of a tube. The dimensions of each prosthesis were $19.7 \mathrm{~mm}$ internal diameter, $23 \mathrm{~mm}$ external diameter, and $30 \mathrm{~mm}$ long. A ring of solid titanium reinforced the central part of the prosthesis to confer more solidity. At each end of the prosthesis, six $1 \mathrm{~mm}$-diameter holes allowed for sutures between the prosthesis and the tracheal extremities.

PROTiP also manufactures solid Ti40 titanium prostheses that act as the inner liner of the porous titanium tracheal prostheses. We used the solid prostheses to prevent the proliferation of exophytic endoluminal tissue and permit the use of mucous grafts to cover the endoluminal surface of the porous titanium prostheses. Their dimensions were $19.2 \mathrm{~mm}$ external diameter, $0.5 \mathrm{~mm}$ thick, and $50 \mathrm{~mm}$ long.

When we replaced a tracheal segment, we used silicone tubes (stents) for the endoluminal calibration of the porous titanium prostheses. Their hardness was 50 Shore A; they were $80 \mathrm{~mm}$ long with an internal diameter of $19 \mathrm{~mm}$. 


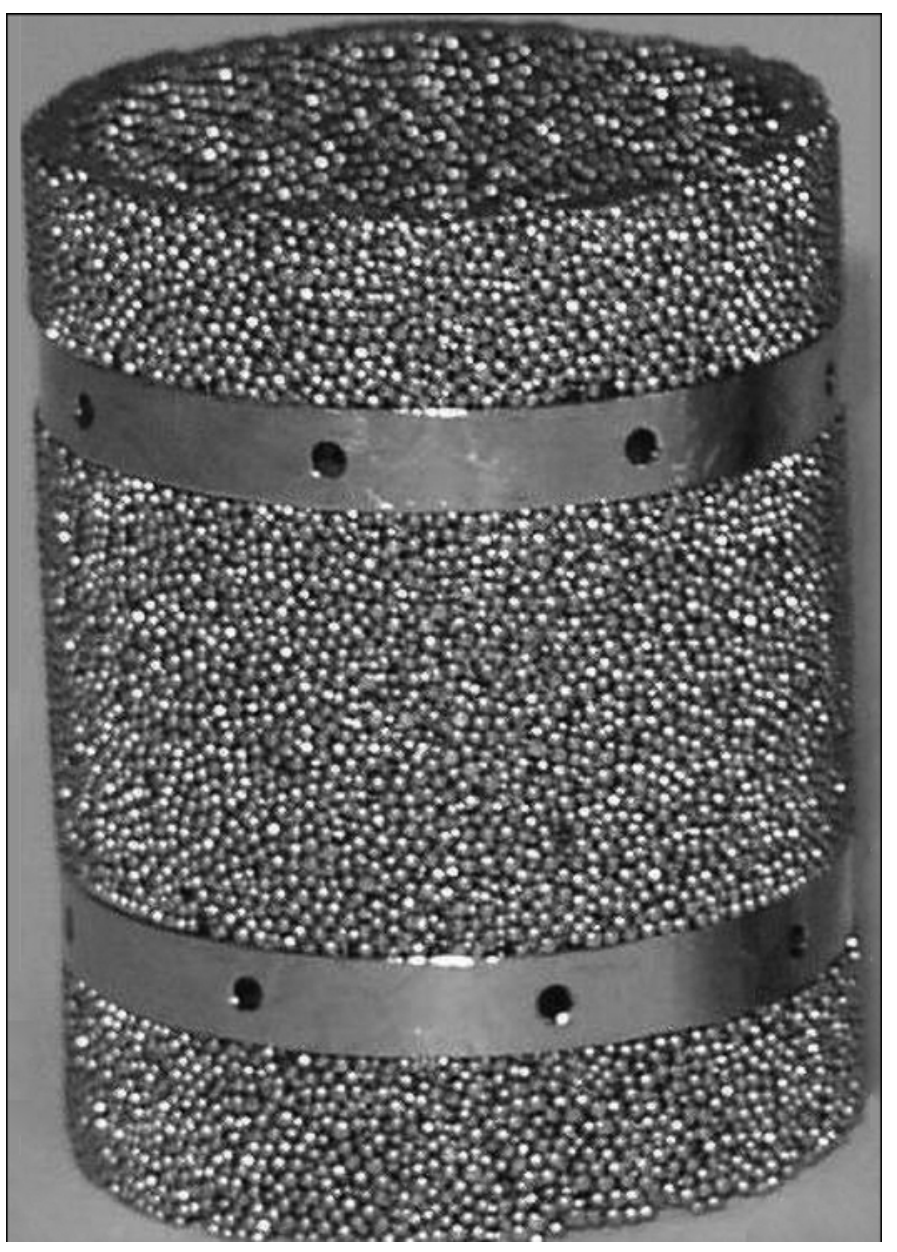

Figure 1.

Tracheal prosthesis made of porous titanium with double ring of bulk titanium.

They protruded from each end of the prosthesis by $25 \mathrm{~mm}$, thus protecting the tracheo-prosthetic anastomoses. The prosthetics and silicone tubes were sterilized by autoclaving before implantation.

\section{Animal Model}

For this study, we used sheep $(n=11)$ between the ages of 4 and 11 months and weighing an average of $60 \mathrm{~kg}$. Each animal received care in compliance with the Guide for the Care and Use of Laboratory Animals [7]. The implantation and explantation operations were performed at the Institut Mutualiste Monsouris (IMM) in Paris, France.

\section{Anesthesia}

All surgeries were performed under general anesthesia. After providing ketamine $(6 \mathrm{mg} / \mathrm{kg})$ as intramuscular premedication, we induced anesthesia by intravenous administration of sodium thiopental $(10 \mathrm{mg} / \mathrm{kg})$. We maintained anesthesia by ventilation with inhaled halothane (1\%-2\% isoflurane and $100 \%$ oxygen) after endotracheal intubation. An intravenous injection of cefamandol (15 mg/kg) provided antibiotic prophylaxis for the entire surgical procedure. We maintained postoperative analgesia by intramuscular injections of morphine salt $(0.2 \mathrm{mg} / \mathrm{kg})$ for 5 days.

\section{Surgical Techniques}

We performed five different implantation methods: Implantation method 1 (IM1) $(n=5)$ involved direct implantation of the porous titanium prosthesis with an endoprosthetic silicone tube in place of a tracheal segment. With the animal in the supine position after a vertical midline cervicotomy, the infrahyoid muscles were separated from the tracheal axis. After dissecting to the level of the thyroid, we resected a tracheal segment of six to seven rings $(50 \mathrm{~mm})$. Each tracheal extremity was then inserted into the prosthesis, which replaced the defect. A silicone tube was placed in the titanium prosthesis and sutured to the prosthesis with one proximal and one distal stitch (vicryl 2.0). For animal 1, the stent-prosthesis suture was not performed. The porous titanium prosthesis and the tracheal extremities were joined by eight proximal and distal sutures (prolene 2.0). Before closing the skin and the subcutaneous layers without drainage, we performed a myoplasty to cover the prosthesis and limit possible peritracheal leakage.

IM2 $(n=2)$ involved Intramuscular placement of a porous titanium prosthesis and a solid titanium endoprosthesis in the quadriceps. After positioning the animal in the lateral decubitus position, we incised the skin on the lateral surface of the thigh vertically. The prosthesis and an endoprosthetic tube of solid titanium were then housed in a cavity created in the quadriceps muscles. We then sutured the skin and subcutaneous layers with separated stitches of vicryl 2.0. The goal of this technique was to study the prostheses' colonization by muscular tissue in an aseptic environment.

In IM3 $(n=2)$, implantation was peformed in two steps:

1. Conditioning of the prosthesis with a solid titanium endoprosthetic tube under the infrahyoid muscles (day 0).

2. Implantation of the prosthesis to replace a resected tracheal segment (day 25). 
After placing the animal in the supine position, we performed a cervicotomy with a midline vertical incision, allowing access to the infrahyoid muscles, which we separated from the tracheal axis. We placed the prosthesis between the infrahyoid muscles and the trachea and then inserted a solid titanium tube into the porous titanium prosthesis. The skin and subcutaneous layers were then closed with separated stitches of vicryl 2.0.

On day 25 after implantation, we retracted the solid titanium tube through the same incision and laterally transposed the porous titanium prosthesis, replacing a tracheal segment of $5 \mathrm{~cm}$ (6-7 rings), which we resected during the same surgery. We inserted the proximal and distal tracheal extremities into the titanium prosthesis and joined the junctions of the prosthesis and the tracheal extremities with 14 sutures of prolene 2.0. Before the sequential closing of the layers, we performed a myoplasty to cover the incision.

In IM4 $(n=1)$, implantation was performed in two steps:

1. Conditioning of the prosthesis with a solid titanium endoprosthetic tube under the infrahyoid muscles (day 0).

2. Implantation of the prosthesis with a silicone tube to replace a resected tracheal segment (day 25). We repeated the surgical technique described in IM3 but with the additional insertion of a silicone tube. We placed the tube, extending beyond the ends of the prosthesis, inside the porous titanium prosthesis and sutured to it with a proximal and a distal suture (vicryl 2.0) during implantation to replace a tracheal segment.

In IM5 $(n=1)$, Implantation was performed in three steps:

1. Conditioning of the porous titanium prosthesis with a solid titanium endoprosthetic tube under the infrahyoid muscles (day 0) for 60 days.

2. Grafting mucosal epithelium from the animal's buccal floor onto the endoluminal surface of the prosthesis. We placed a tube of solid titanium as an endoprosthetic to apply the graft (day 60). Together, the prosthesis and its graft were conditioned for 21 more days.

3. We removed the solid titanium tube, then implanted the prosthesis and the endoluminal epithelial graft with an endoprosthetic silicone calibration tube in place of a tracheal segment (day 81).

After placing the animal in the supine position, we performed a cervicotomy via a midline vertical incision to allow access to the infrahyoid muscles, which we separated from the tracheal axis. We positioned the prosthesis between the infrahyoid muscles and the trachea with a solid titanium endoprosthetic tube. We then closed the skin and subcutaneous surfaces with separated stitches of vicryl 2.0 (day 0).

After 60 days, we took a mucosal epithelium sample from the floor of the ewe's mouth. We reopened the cervical incision and used the buccal mucosa to cover the endoluminal surface of the porous titanium prosthesis (day 60) after we removed the solid titanium tube placed on day 0 . We inserted another solid titanium tube (with a smaller diameter than that of the porous titanium prosthesis) into this prosthesis to secure the epithelium to the endoluminal surface. The prosthesis, covered with the epithelium on the endoluminal side, was conditioned for 21 days.

After 21 days (day 81), we reopened the cervical cutaneous incision and laterally transposed the prosthesis, with its endoluminal surface covered with buccal epithelium, to replace a $5 \mathrm{~cm}$ tracheal segment (6-7 rings), which we resected during the same operation. We removed the solid titanium tube and replaced it with a silicone tube (placed inside the porous titanium prosthesis) that was sutured to the prosthesis with one proximal and one distal stitch (vicryl 2.0). Before closing the skin and subcutaneous tissue, we performed a myoplasty to improve coverage and limit future leakage.

\section{Clinical and Endoscopic Follow-Up}

We performed a daily clinical follow-up. We recorded data regarding the general well-being of the animals and their weight throughout the experiment. We performed endoscopic follow-up (with archival films and photographs) under general anesthesia weekly during the first month after tracheal-segment prosthesis implantation, then every 2 weeks, to evaluate the endoluminal diameter of the prosthesis and the colonization tissue. Endoscopic evaluations were also performed in cases of dyspnea and before scheduled euthanasia.

\section{Histological Analysis}

After an observation period ranging from 1 to 14 months, the sheep were euthanized with a mixture of propofol and potassium chloride. We performed a block resection, including surrounding tissues as well as the prosthesis. The tissue was then fixed in 10 percent formaldehyde for preservation, dehydrated with an alcohol 
gradient, and embedded in paraffin. We distinguished the prostheses from surrounding tissue with radiography. We obtained $5 \mu \mathrm{m}$-thick sections (equivalent to a horizontal section under the solid titanium ring and two vertical sections above and below the ring) with an HM 350 microtome, stained them with hematoxylin-eosin-safran, and viewed them under an optical microscope (Nikon Eclipse E 600, Nikon Instruments, Inc; Melville, New York). We analyzed the tissue integration of the prosthesis, local inflammatory reactions, the endoluminal colonization of the epithelium, and the internal diameter. We performed histological analyses at the IMM and at Biomatech (Chasse sur Rhone, France).

\section{RESULTS}

\section{Clinical Results}

The results are reported in the Table. The surgeries and the immediate postoperative care had no complications.

In IM1 $(n=5)$, the endoscopic test performed at day 6 on animal 1 showed proximal migration of the stent; it had not been fixed. We removed the silicone tube on day 13 by endoscopy. The distal anastomosis (the area not in contact with the stent) exhibited stenosis, which subsequently worsened until the animal's death on day 32. In contrast, the proximal anastomosis had been calibrated by the stent and remained nonstenotic. This finding emphasizes the importance of initial calibration, because inflammation and the formation of granular tissue are typically greatest during the first weeks.

The regular endoscopic tests performed on animal 2 showed a large endoprosthetic lumen with good application of the silicone tube (Figure 2). The stent was removed on day 55, with no stenosis found in later endoscopic tests. The animal was euthanized on day 119, as initially planned.

For animal 3, we removed the stent on day 143, after a surveillance period and a wait for the endoscopic tests to stop detecting stenosis. Later endoscopic follow-ups showed an endoprosthetic lumen without any narrowing. The sheep was euthanized on day 172.

For animal 4, we never removed the stent. The sheep was generally in good shape throughout the study; the endoscopic tests show no stenosis. The animal was euthanized on day 419.
Animal 5 showed a favorable clinical and endoscopic profile similar to that of animal 4 . We removed the stent on day 273, and the animal was euthanized on day 362.

In IM2 $(n=2)$, we placed prostheses in both thighs of animal 6 . The animal was euthanized on day 400 .

We implanted a prosthesis in the right thigh of animal 7 , and the animal was euthanized on day 350 .

The skin conditions of the implantation zones showed no signs of infection. The two sheep were in very good general health throughout the study.

In IM3 ( $n=2)$, animal 8 died on day 31, 6 days after the replacement of a tracheal segment with a prosthesis that had been previously conditioned. Animal 9 died the day after the implantation. Autopsies revealed necrotic stenosis of the tracheo-prosthetic anastomoses.

In IM4 $(n=1)$, animal 10 was in a good general state and was eupneic after we implanted the prosthesis and replaced a tracheal segment. A first endoscopic test on day 28 showed a proximal loop in the silicone tube (stent) (Figure 3). We removed the stent by endoscopy on day 35. After exhibiting an intense stridor, the animal was euthanized. The autopsy revealed an endoprosthetic proximal stenosis in which the stent was not inserted because of plication.

In IM5 ( $n=1)$, the sheep was eupneic and in a good general state throughout the experiment. The animal was euthanized on day 102 as planned, i.e., 3 weeks after prosthesis implantation and tracheal segment replacement. The prosthesis exhibited perfect coverage by the epithelium on the endoluminal surface.

\section{Histological Results}

Only IMs 1, 2, and 5 were subjected to histological analysis because the postsurgical survival of the animals used for IMs 3 and 4 was too brief to provide prosthetic integration results. The analysis focused on the macroscopic and histological evaluation of local tolerance, the depth of tissue integration, the degree of tissue integration at the ends of the implanted tracheal prosthesis, and endoluminal epithelialization.

The histological sections included both the prosthesis and the periprosthetic matrix. The vertical sections allowed us to determine the extent of colonization along the entire length of the prosthesis. The transverse section assessed the regularity of peri-, endo-, and transprosthetic tissue colonization over $360^{\circ}$. 
JRRD, Volume 48, Number 7, 2011

Table.

Clinical and histological results.

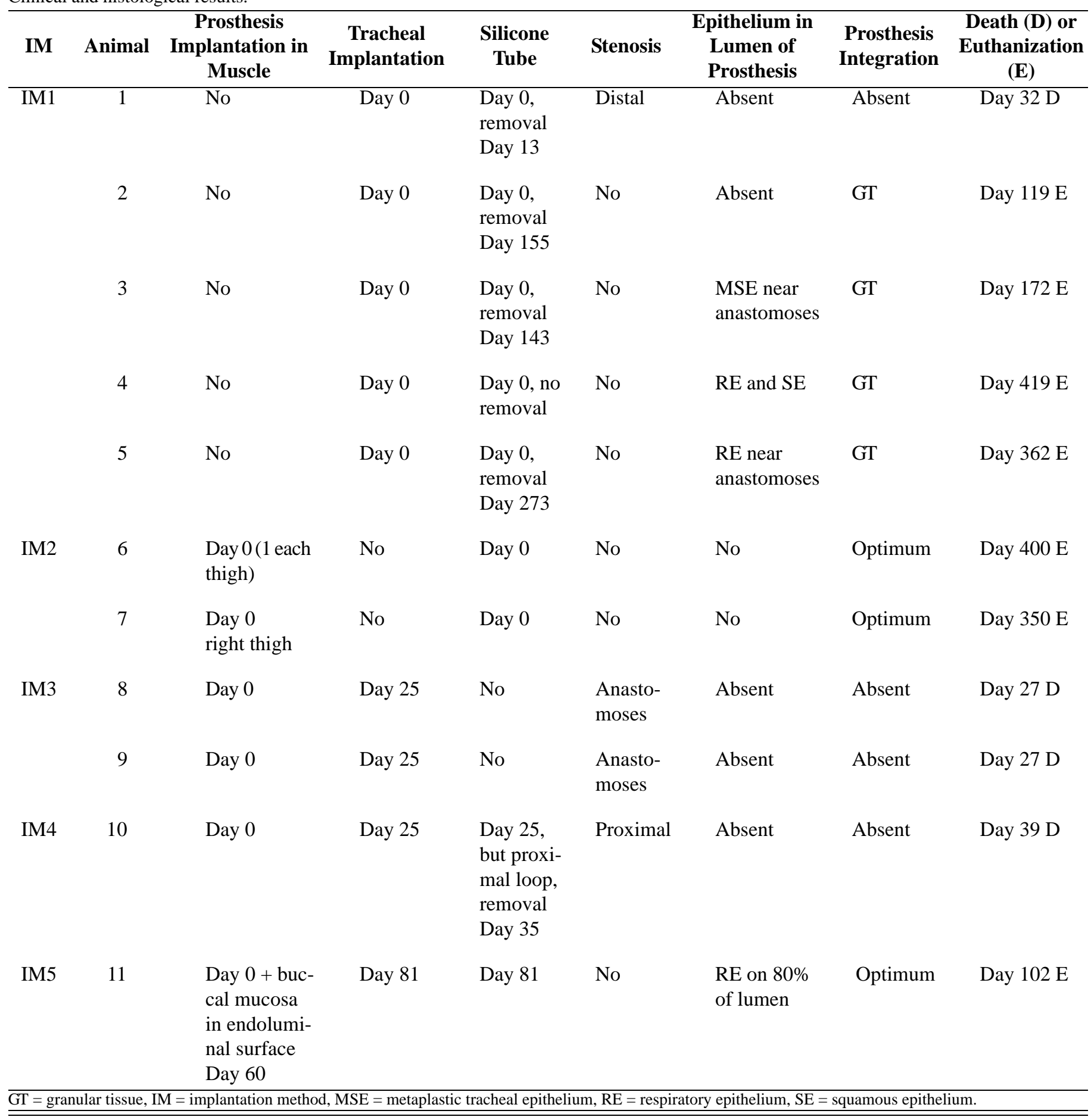

IM1: Histology of Cervical Blocks, Including Prosthesis

Macroscopically, the tracheo-prosthetic junctions were difficult to identify; no loosening of the prosthesis was visible. None of the prostheses showed signs of frac- ture. The prostheses were surrounded by a tissue matrix, with the thickness of the tissue around the prostheses corresponding to about 50 percent of their diameter. A growth of newly formed fibroepithelial tissue opposite 


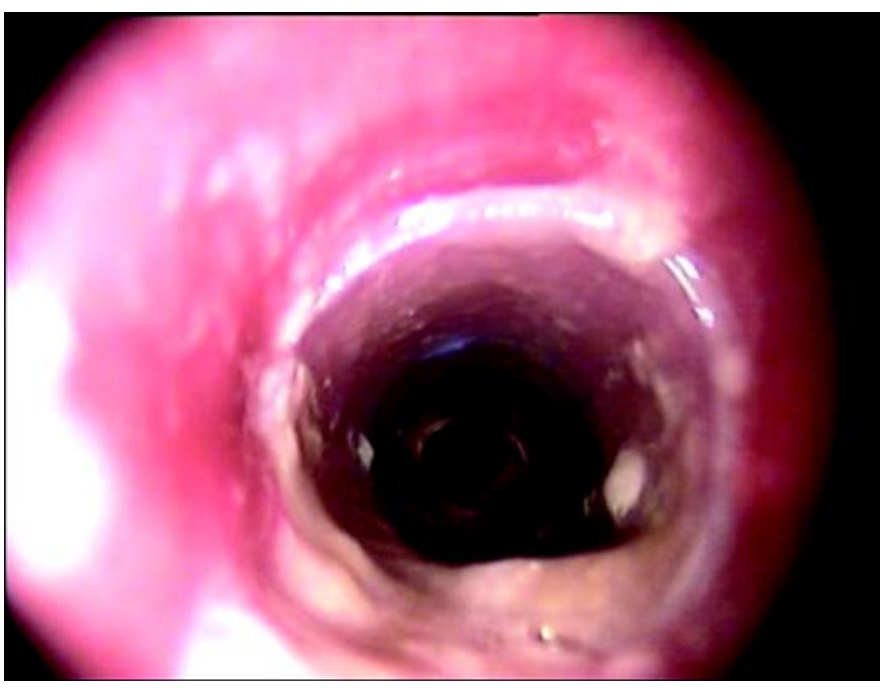

Figure 2.

Endoscopic evaluation of animal 2 on day 55 showed large endoprosthetic lumen with good application of silicon tube.

the juxtaprosthetic endoluminal surface created a partial stenosis without clinical consequence for animals 2 and 3. By contrast, no sign of stenosis on any section was detected in animals 4 and 5 .

Microscopically, the prostheses were encapsulated by dense adventitious fibrovascular tissue continuous with the periprosthetic tracheal tissue. The interface between the external surfaces of the prostheses and the capsule consisted of Malpighian epithelium. The prosthesis spaces defined by the microbeads were mainly filled with cellular debris and inflammatory cells. For animal 3, a metaplastic tracheal epithelium ran alongside the extremities of the endoluminal surface of the prosthesis. For animals 4 and 5, ciliated pseudostratified squamous epithelium, i.e., respiratory epithelium, was visible on the endoluminal surface of the distal third of each prosthesis (Figure 4). Simple squamous epithelium was present on the central third of the endoluminal surface of animal 4's prosthesis (Figure 5).

\section{IM2: Histology of Prosthesis Implanted in Muscular Tissue}

Each of the three prostheses was included in the neighboring muscle. The pores between the microbeads were infiltrated by mature connective tissue composed of fibroblasts (Figure 6), collagen, and microvasculature, with a very small number of multinucleated giant cells. These results confirmed the very good integration and

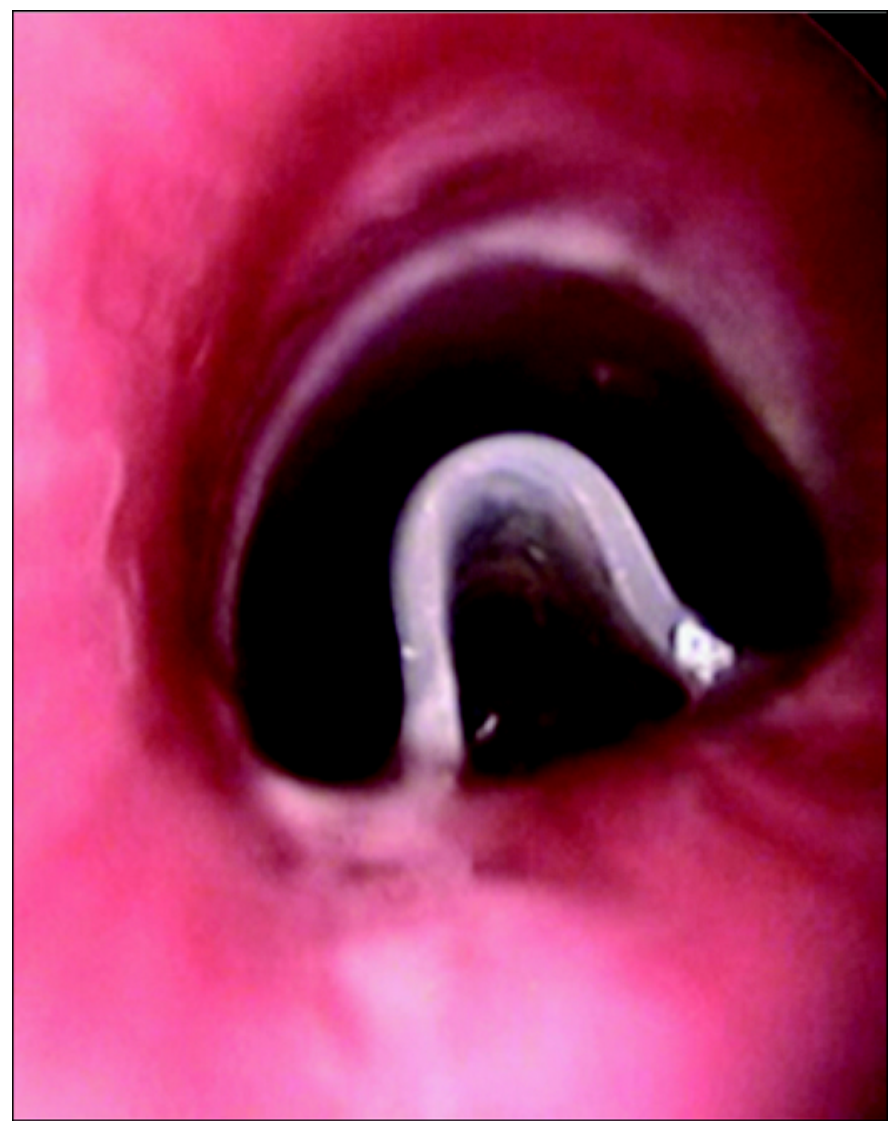

Figure 3.

Endoscopic evaluation of animal 10 on day 28 showed proximal look of silicon tube.

optimal local tolerance of the intramuscularly implanted prostheses.

\section{IM5: Histology of Cervical Block, Including Prosthesis}

Macroscopically, the junctions of the trachea and the porous titanium prosthesis were difficult to identify, and the prosthesis showed no signs of fracture. The endoprosthetic lumen was large, reduced by a maximum of onethird of its diameter relative to the diameter of the naked porous titanium prosthesis.

The endoprosthetic surface was covered with squamous epithelium (about $80 \%$ of the total surface) (Figure 7), except for some scattered fields (about $20 \%$ of the surface) devoid of epithelium and filled with inflammatory tissue (polynuclear neutrophils and macrophages).

Below this endoluminal squamous epithelium was supportive fibrous tissue with numerous capillary vessels. This vascularized fibrous tissue extended toward the 


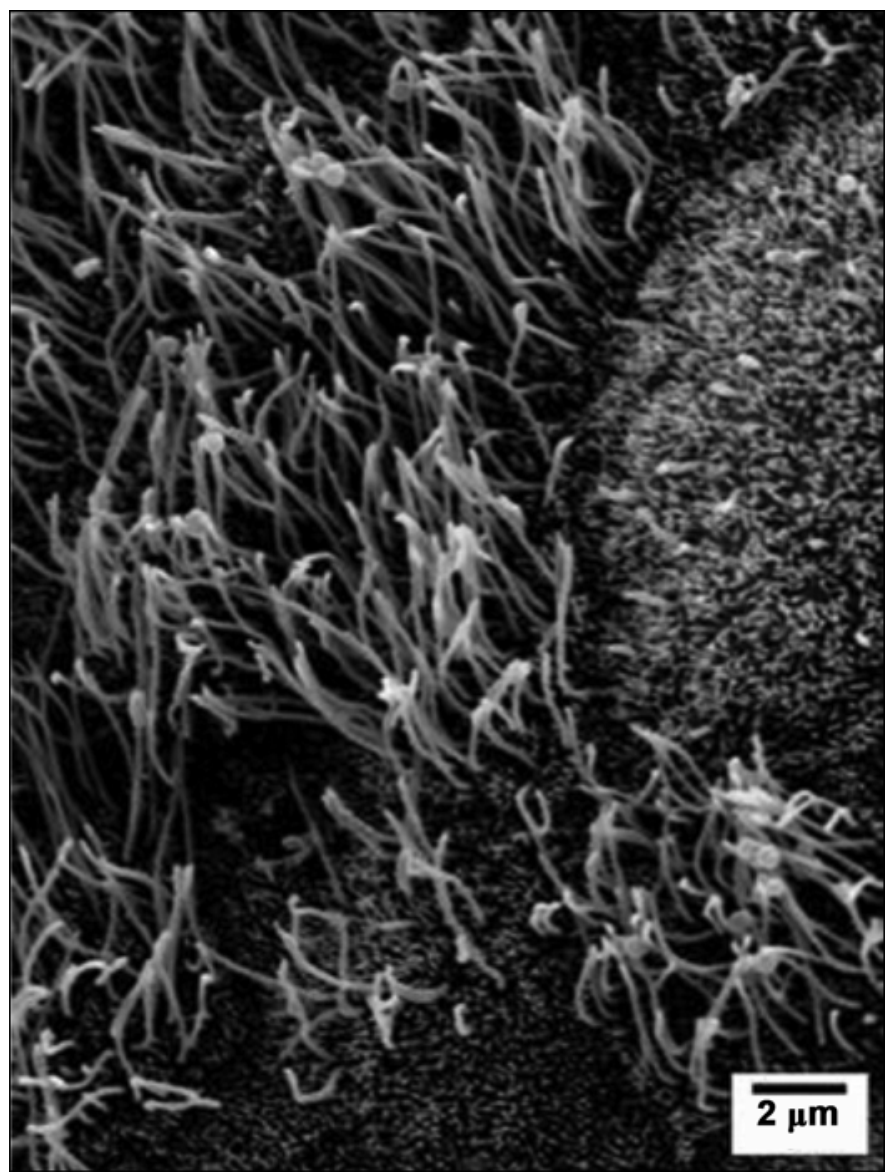

Figure 4.

Scanning electron microscopy view of distal third of animal 5's prosthesis. Ciliated epithelium, i.e., respiratory epithelium, is present.

periphery and filled in the pores of the titanium prosthesis (Figure 8). The presence of fibrovascular tissue between the titanium microbeads confirmed that the prosthesis integrated quite well.

\section{DISCUSSION}

Total laryngectomy is routinely practiced in cases of advanced pharyngolaryngeal cancer, when partial surgery or an organ preservation protocol (radiochemotherapy) is impossible or likely to fail. Removal of the larynx, with the loss of phonatory function and the need for a permanent tracheostomy, has dramatic consequences for the patient. The majority of worldwide research in the field is focused on vocal rehabilitation, but a few studies have attempted to abolish the tracheostomy opening, which

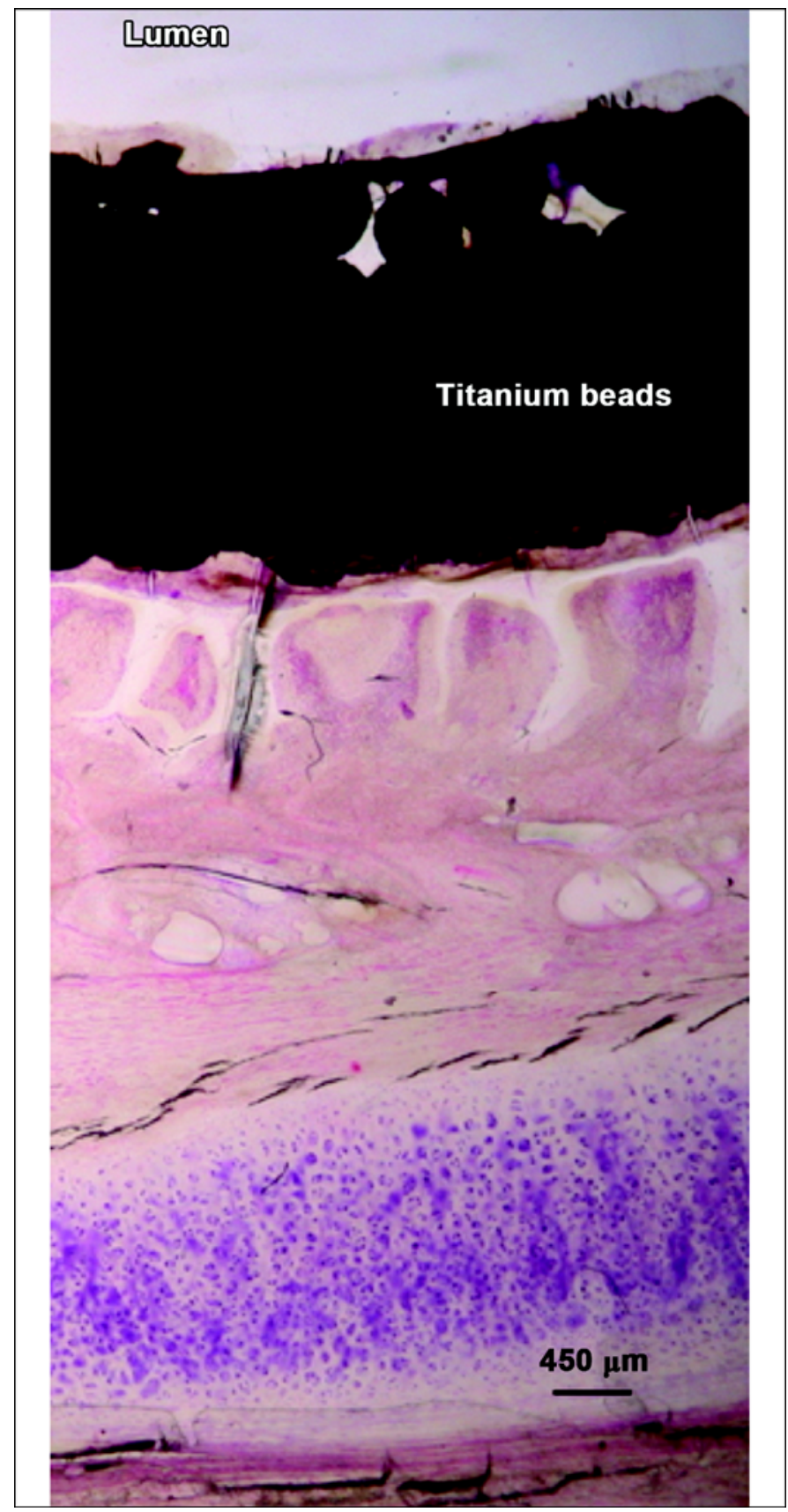

Figure 5.

Histological examination of central third of animal 4's tracheal prosthesis. Lumen is covered with simple squamous epithelium (hematoxylin-eosin-saffron).

would require reestablishing a common passage between the respiratory and digestive tracts. Our work focused on designing an implantable laryngeal prosthesis that would combine a rigid, immovable structure with a biofunctional, 


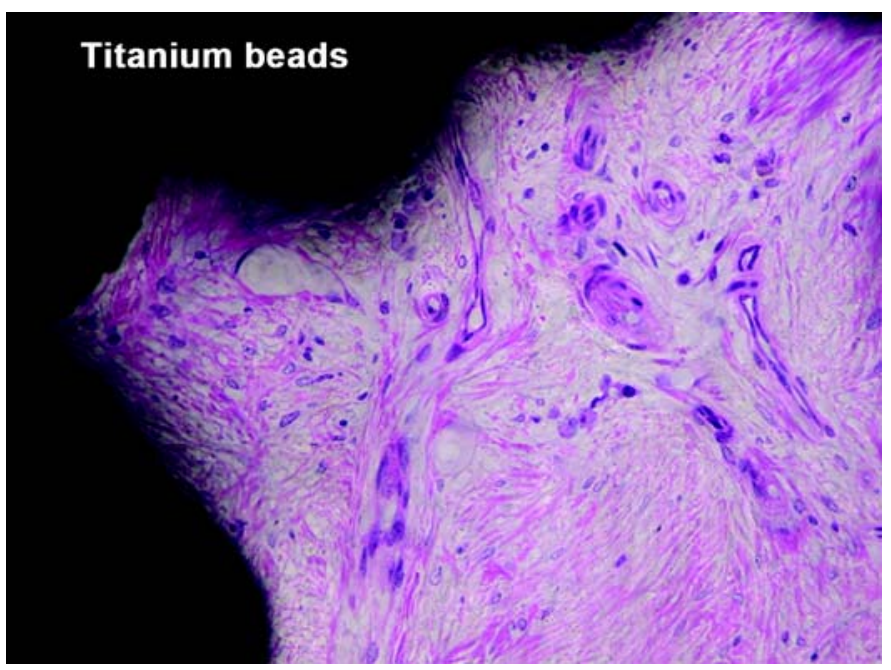

Figure 6.

Microscopic view of animal 6's prosthesis after muscle implantation. Pores between beads are infiltrated by mature connective tissue composed of fibroblasts (hematoxylin-eosin-saffron).

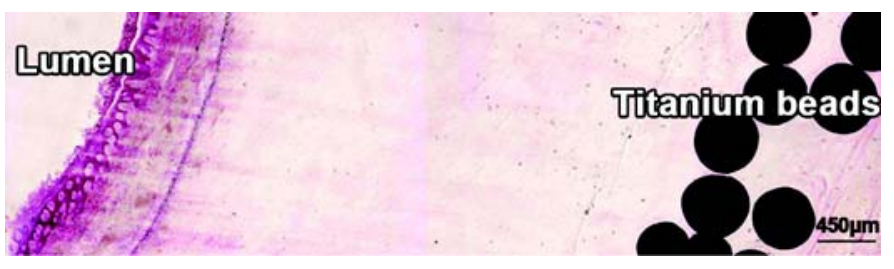

Figure 7.

Histological examination of middle of animal 11's prosthesis. Squamous epithelium is present in lumen and pores are infiltrated by fibrovascular tissues (hematoxylin-eosin-saffron).

movable structure (the upper valve would synchronize the respiratory and digestive functions). The rigid structure was designed to lengthen the proximal trachea after a total laryngectomy.

The search for an ideal tracheal substitute has been the focus of many studies for more than 50 years. Several other teams have addressed the same problem of replacing a tracheal segment, but with a goal other than creating an artificial larynx. In cases of tumoral or traumatic tracheal lesions extending along more than half of the trachea in adults (or one-third in children), direct anastomosis is impossible to perform, even with maximal tracheal mobilization. Extended tracheal resections remain an unresolved surgical problem during reconstruction. Numerous replacement materials have been tested [8-10] on animal models, including synthetic prostheses [3-

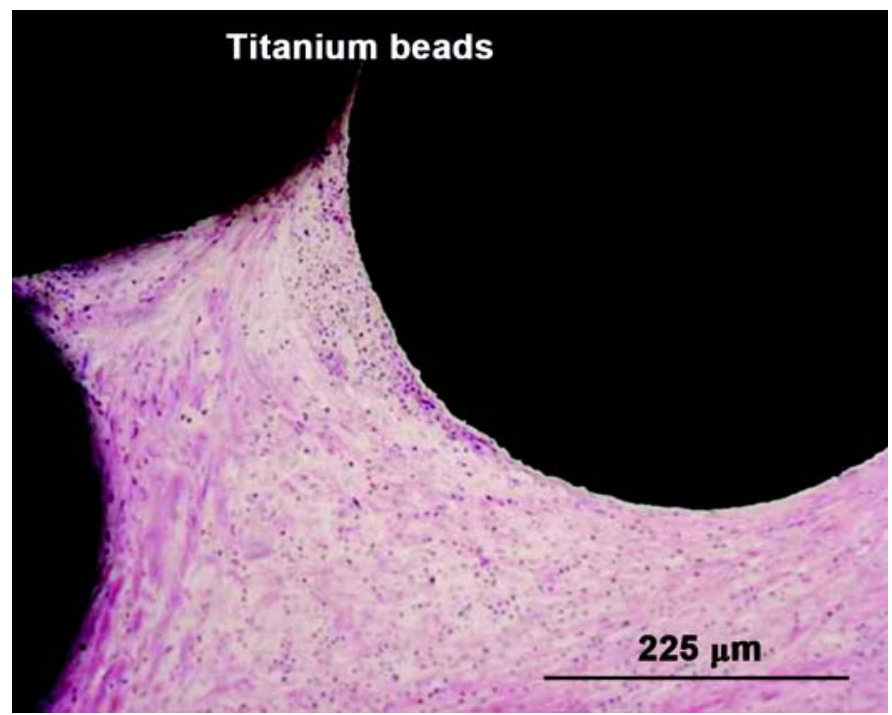

Figure 8.

Microscopic view of animal 11's prosthesis. Vascularized fibrous tissue filled in pores of titanium prosthesis (hematoxylin-eosin-saffron).

4,11], tracheal homo- and allografts [12-13], tissue autoand allotransplantations [14-23], and cultured cell seeded implants [24-25]. Martinod et al. used an aortic allograft linked to a silicone prosthesis as a tracheal substitute [14-19]. The initial results, obtained in sheep, were spectacular: the silicone tube was removed after 6 months, and the aortic tissue was transformed into a real trachea composed of cartilage organized in rings and covered with a mucociliary epithelium. The preliminary results in humans were contradictory. Of six human patients, four survived after an average follow-up of 34 months and none benefited from removal of the silicone tube [26]. In addition, the process of aortic tissue reorganization into a cartilaginous structure seems delayed in humans because it was only partially complete after 34 months.

Despite the undeniable value of these results, the use of this aortic graft may not be suitable for laryngeal reconstruction. Given its initial flaccidity and the impossibility of removing the silicone prosthesis in humans, this graft cannot be attached to an upper valve that would replace the laryngeal sphincter.

The previous work of our team led to the development of a biomaterial consisting of an assembly of titanium microbeads [2-4]. This biomaterial meets several criteria: (1) being mechanically rigid, it provides support when it replaces tracheal rings; (2) being inert, it limits detrimental cellular reactions; and (3) it resists aggressive environments such as those of the upper aerodigestive 
tracts. An implant meant to substitute for laryngeal or tracheal cartilages must have biofunctional properties similar to those of the respiratory tree. The implant must be perfectly integrated, i.e., perfectly colonized by the periprosthetic tissue and entirely covered by a respiratory epithelium on the endoprosthetic surface.

The implantations of uncoated titanium tracheal prostheses in rats [2-3] offered encouraging results that allowed us to proceed to implanting tracheal prostheses in sheep [4]. The first implantations performed in ewes showed good immediate tolerance; no postsurgical complications occurred. The complications and mortality occurred secondarily, after infection and prosthetic obstruction. The implants were obstructed by a thickening of the tracheal stumps via inflammatory granulation tissue. The granuloma caused bronchial stasis, which gave rise to pulmonary infections and the formation of casts or mucus plugs. Necrotic zones at the junctions of the tracheal extremities and the prosthesis were also present in some sheep. Also, the colonization of the pores of the prosthesis remained poor. The surgical protocol used in that study (the one-step implantation of a titanium prosthesis in place of a tracheal segment without a stent) was considered unsatisfactory.

Throughout our studies with porous titanium prostheses, we observed no loosening, fractures, or rejections of prostheses. These results confirm our experience with mandibular prostheses made of porous titanium in humans [27]: although the mechanical pressure was high, none of these problems occurred.

The study described here focused on the implantation of titanium prostheses in five groups of sheep. Five different surgical protocols were performed. Our goal was to establish, by comparing the results from each group, an ideal surgical technique for implanting a porous titanium tracheal prosthesis in large animals. The main criteria necessary for a biofunctional tracheal prosthesis are as follows: maintenance of a large endoprosthetic lumen, colonization of the prosthetic pores by fibrous connective tissue, and development of respiratory epithelium covering the entire endoluminal surface of the prosthesis.

In IM1 $(n=5)$, the porous titanium prosthesis was implanted with a silicone endoprosthetic calibration tube directly in place of a tracheal segment. Long survivals were observed in this group (euthanizations occurred on days 119,172 , 362, and 419). Necrotic zones were almost nonexistent; the same was true for stenotic zones (which were completely absent from animals 4 and 5). The sili- cone tube calibrated the size of the airways [27] and prevented the formation of granulation tissue, which from our experience is a major cause of fatal airway stenosis [4]. Granulation tissue stents the proximal and distal anastomoses while preventing the penetration of mucus in the luminal pores toward the periphery of the prosthesis. The stent acts as a protective barrier that maintains the respiratory lumen while counterbalancing intrinsic and extrinsic pressures, thus acting as a splint. Makris and Marquette emphasized these findings when treating tracheobronchial obstructions with a stent and reported the silicone tube stent's superiority over the metallic stent for calibrating the airways [28]. An endoprosthetic epithelium was detected in IM1; it was identified in animal 3 (euthanized on day 172) in the distal extremities of the endoluminal surface of the prosthesis as a metaplastic tracheal epithelium. For animals 4 (euthanized on day 419) and 5 (euthanized on day 372), a respiratory (pseudostratified, ciliated) squamous epithelium was identified endoprosthetically. This epithelium covered the proximal and distal thirds of the endoluminal surface of the prostheses. For animal 4, the central third of the prosthesis was colonized by a simple squamous epithelium. These histological results suggest that the respiratory epithelium proliferated from the tracheal extremities that form the junction with the prosthesis; i.e., it was a continuous epithelialization. The presence of an endoprosthetic squamous epithelium in IM1 confirmed that the stent did not hinder respiratory epithelium proliferation. On the contrary, by preventing tracheal secretions from penetrating through the pores, from the lumen toward the exoluminal part of the prosthesis, the stent allowed the epithelial cells to proliferate without being subjected to the flow of mucus.

However, in IM1, the colonization of the prosthesis pores remained insufficient. A matrix composed of thick, dense granulomatous and fibrous connective tissue surrounded the prostheses and kept them aligned in the tracheal axis, allowing them to perform their functions; however, the pores of the prostheses were only filled with inflammatory cells and cellular debris and not with connective tissue, as had been expected.

To determine whether the problems of cellular colonization of the pores were linked to the premature filling of the pores with mucus, we performed IM2. The goal of IM2 $(n=2)$ was to test only the colonization of the prosthesis pores in an aseptic environment with simple muscular contact with the periphery of the prosthesis. In 
this group, the porous titanium prostheses (with an endoprosthetic tube made of solid titanium) were implanted in the quadriceps muscles. The histological analysis of these implants showed perfect integration. All the pores were filled with mature connective tissue consisting of fibroblasts that formed a bed capable of sustaining the proliferation of a viable epithelium to cover the endoprosthetic surface.

In IM3 $(n=2)$, we implanted prostheses by replacing a tracheal segment without the silicone tube after conditioning the prostheses in the infrahyoid muscles for 25 days. The animals used in this procedure died prematurely (days 27 and 29). The low survival rate of the sheep was directly linked to endoprosthetic stenosis or to necrotic junction zones. Furthermore, the bodies of the prostheses were covered with endoluminal mucus without colonization. No stenosis at the level of the prosthesis body was documented; only the tracheo-prosthetic anastomoses were involved. The prior conditioning and colonization of prostheses did not warrant bypassing the use of a stent. These results support the hypothesis that the silicone tube is necessary to protect the respiratory lumen and to stent the junction zones. The role of the silicone tube as a splint is essential, even with the improved impermeability of the conditioned prosthesis.

In accordance with these results, a sheep was implanted with a preconditioned prosthesis made of porous titanium and with a silicone tube. In IM4, we replaced a tracheal segment with a porous titanium prosthesis conditioned for 25 days and a silicone tube. The endoscopic tests demonstrated a proximal plication of the silicone tube, requiring its early removal. The animal was euthanized 4 days after the stent was removed because of inspiratory dyspnea associated with proximal endoprosthetic stenosis by granulation tissue (where the stent could not function because of plication). This finding confirmed the importance of correctly applying the stent on the endoluminal surface.

Endoluminal inflammation, likely promoting stenosis, was found in all the IMs when the stent was removed prematurely. Because the respiratory epithelium migrates very slowly (only animal 4, euthanized late at 419 days, showed total colonization of the endoprosthetic surface), accelerating its proliferation seems necessary. Performing tracheal reconstructions with a prosthesis whose endoluminal surface is pre-epithelialized (i.e., immediately functional) would eliminate the wait for the slow epithelial migration from the tracheal extremities and the classic inflammation associated with healing processes. We also performed IM5 to accelerate endoprosthetic healing by directly grafting from the oral mucosa to the lumen of the porous titanium prosthesis while using the initial technique of conditioning the prosthesis and implanting it with a silicone tube in place of a tracheal segment.

In terms of the quasitotal and immediate coverage of the endolumenal surface of the prosthesis by a squamous epithelium, as well as the absence of endoprosthetic stenosis, the results were very satisfactory. The initial conditioning provided fibrovascular supportive tissue (filling in the pores of the prosthesis) to the endoluminal epithelium, which covered more than 80 percent of the endoprosthetic surface by 21 days after the prosthesis was implanted in place of a tracheal fragment.

The method used in this last group appears to be the only truly effective one. Only one sheep was used for this technique, for only a short-term study (it was euthanized 21 days after the tracheal prosthesis was implanted). A long-term study using this method of implantation is essential for evaluating the tolerance, survival, and histological development of the endoluminal epithelial graft. Future studies should include the three following steps: (1) conditioning the prosthesis, (2) grafting an endoluminal epithelium, and (3) implanting the prosthesis with a silicone stenting tube in place of a tracheal segment.

The step of directly grafting epithelium endoprosthetically on a previously cultured prosthesis makes the tracheal prosthesis immediately functional. This graft can originate from mucosal epithelium taken endobuccally (as in IM5). Another approach, which is currently being developed, colonizes the endoluminal surface of the prosthesis in vitro with ciliated cells, thus directly creating a respiratory epithelium before the prosthesis was implanted in place of a tracheal segment (collaboration with Institut National de la Santé et de la Recherche Médicale Unit 903, Reims, France) [29-30].

\section{CONCLUSIONS}

Our research aims to preserve a certain quality of life for laryngectomees by abolishing the mutilating consequences of the loss of the larynx, i.e., tracheostomy and complete separation of the respiratory and digestive functions. For laryngeal function to be recreated, the trachea must first be lengthened with a hollow, integrated structure that is vascularized by the surrounding tissues. Numerous 
authors have considered the use of synthetic prostheses to replace a tracheal segment an inevitable failure, given the lack of graft epithelialization and/or the presence of tracheal obstruction or prosthetic migration [18,22].

Our team focused its research on replacing a tracheal segment with porous titanium Ti40 biomaterial. This sheep study has shown the prostheses' partial endoprosthetic colonization by respiratory epithelium and its functionality when replacing a tracheal segment, as authenticated by long survival of the ewes. However, the optimal integration of the prosthesis and colonization by a respiratory epithelium on the whole endoprosthetic surface remains to be achieved.

By comparing different methods of implantation, this study yields three main results that need to be considered to make a porous titanium prosthesis completely biofunctional. Stenoses in the junctions of the prosthesis and tracheal extremities, which cause premature death, were absent when an endoprosthetic silicone tube was used. The colonization of the pores of the porous titanium prosthesis was improved by the initial implantation of the prosthesis in a muscle, and the endoprosthetic epithelialization was accelerated by the grafting of amucosal tissue in the prosthetic lumen. These findings led us to establish an optimal three-step surgical protocol: (1) preconditioning the prosthesis, (2) grafting endoluminal epithelium, and (3) tracheal implantation, using a silicone tube for calibration. To reinforce the hypotheses presented in this study, a long-term study of tracheal prosthetics implanted according to this technique will be performed soon.

\section{ACKNOWLEDGMENTS}

\author{
Author Contributions: \\ Study concept and design: P. Schultz, C. Debry, A. Dupret-Bories. \\ Technical surgery: P. Schultz, C. Debry, A. Dupret-Bories. \\ Acquisition of data: A. Dupret-Bories, P. Schultz, C. Debry. \\ Analysis and interpretation of data: A. Dupret-Bories, P. Schultz, \\ C. Debry. \\ Drafting and revision of manuscript: A. Dupret-Bories, P. Schultz, \\ C. Debry, N. E. Vrana, P. Lavalle, D. Vautier. \\ Obtained funding: C. Debry, P. Lavalle, D. Vautier.
}

Financial Disclosures: The authors have declared that no competing interests exist.

Funding/Support: This material was based on work supported by the Réseau National des Technologies de Santé de l'Agence Nationale de la Recherche [French National Research Agency], PROTimplant Project.

Additional Contributions: We thank PROTiP for providing the tracheal prostheses.
Institutional Review: This study was approved by the Comité d'Ethique de l'IMM Recherche [Ethics Commitee of IMM Research].

\section{REFERENCES}

1. Babin E, Edy E, Béquignon A, Hitier M. [Personal and social identity transformations that occur over time among patients with total laryngectomy]. J Otolaryngol Head Neck Surg. 2008;37(4):495-501. French. [PMID: 19128582]

2. Schultz P, Vautier D, Chluba J, Marcellin L, Debry C. Survival analysis of rats implanted with porous titanium tracheal prosthesis. Ann Thorac Surg. 2002;73(6):1747-51. [PMID: 12078764$]$ DOI:10.1016/S0003-4975(02)03569-5

3. Schultz P, Vautier D, Egles C, Debry C. Experimental study of a porous rat tracheal prosthesis made of T40: Long-term survival analysis. Eur Arch Otorhinolaryngol. 2004;261(9): 484-88. [PMID: 14655018]

DOI:10.1007/s00405-003-0717-5

4. Schultz P, Vautier D, Charpiot A, Lavalle P, Debry C. Development of tracheal prostheses made of porous titanium: A study on sheep. Eur Arch Otorhinolaryngol. 2007; 264(4):433-38. [PMID: 17123095] DOI:10.1007/s00405-006-0195-7

5. Guillemot F, Porté MC, Labrugère C, Baquey Ch. Ti4+ to Ti3+ conversion of $\mathrm{TiO} 2$ uppermost layer by low-temperature vacuum annealing: Interest for titanium biomedical applications. J Colloid Interface Sci. 2002;255(1):75-78.

[PMID: 12702370]

DOI:10.1006/jcis.2002.8623

6. Ten Hallers EJ, Rakhorst G, Marres HA, Jansen JA, Van Kooten TG, Schutte HK, Van Loop JP, Van den Houwen EB, Verkerke GJ. Animals model for tracheal research. Biomaterials. 2004;25(9):1533-43. [PMID: 14697856] DOI:10.1016/S0142-9612(03)00500-3

7. Committee for the update of the guide for the care and use of laboratory animals: Institute of Laboratory Animal Resources, National Research Council. Guide for the care and use of laboratory animals. $8^{\text {th }}$ rev. ed. Washington (DC): National Academies Press; 2010.

8. Grillo HC. Development of tracheal surgery: A historical review. Part 1: Techniques of tracheal surgery. Ann Thorac Surg. 2003;75(2):610-19. [PMID: 12607695] DOI:10.1016/S0003-4975(02)04108-5

9. Grillo HC. Development of tracheal surgery: A historical review. Part 2: Treatment of tracheal diseases. Ann Thorac Surg 2003;75(3):1039-47. [PMID: 12645751] DOI:10.1016/S0003-4975(02)04109-7

10. Grillo HC. Tracheal replacement: A critical review. Ann Thorac Surg. 2002;73(6):1995-2004. [PMID: 12078821] DOI:10.1016/S0003-4975(02)03564-6

11. Dodge-Khatami A, Niessen HW, Koole L, Klein MG, Van Gulik TM, De Mol BA. Tracheal replacement in rabbits 
with a new composite silicone-metallic prosthesis. Asian Cardiovasc Thorac Ann. 2003;11(3):245-49. [PMID: 14514557]

12. Neville WE, Bolanowski PJ, Soltanzadeh H. Homograft replacement of the trachea using immunosuppression. J Thorac Cardiovasc Surg. 1976;72(4):596-601. [PMID: 966794$]$

13. Tojo T, Niwaya K, Sawabata N, Kushibe K, Nezu K, Taniguchi S, Kitamura S. Tracheal replacement with cryopreserved tracheal allograft: Experiment in dogs. Ann Thorac Surg. 1998;66(1):209-13. [PMID: 9692466] DOI:10.1016/S0003-4975(98)00270-7

14. Martinod E, Zakine G, Fornes P, Zegdi R, d'Audiffret A, Aupecle B, Goussef N, Azorin J, Chachques JC, Fabiani JN, Carpentier A. [Metaplasia of aortic tissue into tracheal tissue. Surgical perspectives]. C R Acad Sci III. 2000;323(5): 455-60. French.

15. Makris D, Holder-Espinasse M, Wurtz A, Seguin A, Hubert T, Jaillard S, Copin MC, Jashari R, DuterqueCoquillaud M, Martinod E, Marquette CH. Tracheal replacement with cryopreserved allogenic aorta. Chest. 2010; 137(1):60-67. [PMID: 19801581] DOI:10.1378/chest.09-1275

16. Seguin A, Radu D, Holder-Espinasse M, Bruneval $P$, Fialaire-Legendre A, Duterque-Coquillaud M, Carpentier A, Martinod E. Tracheal replacement with cryopreserved, decellularized, or glutaraldehyde-treated aortic allografts. Ann Thorac Surg. 2009;87(3):861-67. [PMID: 19231406] DOI:10.1016/j.athoracsur.2008.11.038

17. Azorin JF, Bertin F, Martinod E, Laskar M. Tracheal replacement with an aortic autograft. Eur J Cardiothorac Surg. 2006;29(2):261-63. [PMID: 16388953] DOI:10.1016/j.ejcts.2005.11.026

18. Martinod E, Azorin J, Carpentier A. [Tracheal replacement: New perspectives]. Rev Mal Respir. 2001;18(6 Pt 1): 639-43. French. [PMID: 11924185]

19. Seguin A, Martinod E, Kambouchner M, Campo GO, Dhote P, Bruneval P, Azorin JF, Carpentier A. Carinal replacement with an aortic allograft. Ann Thorac Surg. 2006; 81(3):1068-74. [PMID: 16488724]

20. Brian E, Gounant V, Fulgencio JP, Milleron B, Bazelly B. [Tracheal replacement using the abdominal aorta. Comments on a case report]. Rev Pneumol Clin. 2007;63(3): 224-29. French. [PMID: 17675946$]$

DOI:10.1016/S0761-8417(07)90127-3

21. Davidson MB, Mustafa K, Girdwood RW. Tracheal replacement with an aortic homograft. Ann Thorac Surg. 2009;88(3):1006-8. [PMID: 19699945]

DOI:10.1016/j.athoracsur.2009.01.044

22. Anoosh F, Hodjati H, Dehghani S, Tanideh N, Kumar PV. Tracheal replacement by autogenous aorta. J Cardiothorac Surg. 2009;4:23. [PMID: 19508714] DOI:10.1186/1749-8090-4-23

23. Go T, Jungebluth P, Baiguero S, Asnaghi A, Martorell J, Ostertag H, Mantero S, Birchall M, Bader A, Macchiarini P. Both epithelial cells and mesenchymal stem cell-derived chondrocytes contribute to the survival of tissue-engineered airway transplants in pigs. J Thorac Cardiovasc Surg. 2010;139(2):437-43. [PMID: 19995663]

DOI:10.1016/j.jtcvs.2009.10.002

24. Macchiarini P, Junglebluth P, Go T, Asnaghi MA, Rees LE, Cogan TA, Dodson A, Martorell J, Bellini S, Parni-gotto PP, Dickinson SC, Hollander AP, Mantero S, Conconi MT, Birchall MA. Clinical transplantation of a tissue-engineered airway. Lancet. 2008;372(9655):2023-30.

[PMID: 19022496]

DOI:10.1016/S0140-6736(08)61598-6

25. Wurtz A, Porte H, Conti M, Dusson C, Desbordes J, Copin MC, Marquette CH. Surgical technique and results of tracheal and carinal replacement with aortic allografts for salivary gland-type carcinoma. J Thorac Cardiovasc Surg. 2010; 140(2):387-93.[PMID: 20381819]

DOI:10.1016/j.jtcvs.2010.01.043

26. Schultz P, Vautier D, Atallah I, Gentine A, Debry C. [Reconstruction of the anterior mandibule using a porous titanium implant: A case report]. Rev Laryngol Otol Rhinol (Bord). 2008;129(3):201-5. French. [PMID: 19694164$]$

27. Ko PJ, Liu CY, Wu YC, Chao YK, Hsieh MJ, Wu CY, Wang CJ, Liu YH, Liu HP. Granulation formation following tracheal stenosis stenting: Influence of stent position. Laryngoscope. 2009;119(12):2331-36. [PMID: 19688861] DOI:10.1002/lary.20615

28. Makris D, Marquette CH. Tracheobronchial stenting and central airway replacement. Curr Opin Pulm Med. 2007; 13(4):278-83. [PMID: 17534173] DOI:10.1097/MCP.0b013e32816b5c3b

29. Kojima K, Bonassar LJ, Roy AK, Mizuno H, Cortiella J, Vacanti CA. A composite tissue-engineered trachea using sheep nasal chondrocyte and epithelial cells. FASEB J. 2003; 17(8):823-28. [PMID: 12724341]

DOI:10.1096/fj.02-0462com

30. Coraux C, Hajj R, Lesimple P, Puchelle E. [Repair and regeneration of the airway epithelium]. Med Sci (Paris). 2005;21(12):1063-69. French. [PMID: 16324647]

Submitted for publication October 6, 2010. Accepted January 28, 2011.

This article and any supplementary material should be cited as follows:

Dupret-Bories A, Schultz P, Vrana NE, Lavalle P, Vautier D, Debry C. Development of surgical protocol for implantation of tracheal prostheses in sheep. J Rehabil Res Dev. 2011;48(7):851-64.

DOI:10.1682/JRRD.2010.10.0194

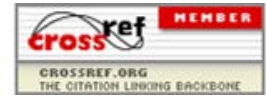


